\title{
Phase II clinical trial of pasireotide long-acting repeatable in patients with metastatic neuroendocrine tumors
}

\author{
M Cives, P L Kunz'1, B Morse, D Coppola, M J Schell, T Campos, P T Nguyen, \\ P Nandoskar', V Khandelwal' ${ }^{1}$ and J R Strosberg \\ Department of Gastrointestinal Oncology, H. Lee Moffitt Cancer Center and Research Institute, \\ 12902 Magnolia Drive, Tampa, Florida 33612, USA \\ 'Department of Medicine, Stanford University School of Medicine, Stanford, California, USA
}

Correspondence should be addressed to J R Strosberg Email

jonathan.strosberg@ moffitt.org

\begin{abstract}
Pasireotide long-acting repeatable (LAR) is a novel somatostatin analog (SSA) with avid binding affinity to somatostatin receptor subtypes 1, 2, $3\left(\mathrm{SSTR}_{1,2,3}\right)$ and 5 (SSTR $)_{5}$. Results from preclinical studies indicate that pasireotide can inhibit neuroendocrine tumor (NET) growth more robustly than octreotide in vitro. This open-label, phase II study assessed the clinical activity of pasireotide in treatment-naïve patients with metastatic grade 1 or 2 NETs. Patients with metastatic pancreatic and extra-pancreatic NETs were treated with pasireotide LAR (60 mg every 4 weeks). Previous systemic therapy, including octreotide and lanreotide, was not permitted. Tumor assessments were performed every 3 months using Response Evaluation Criteria in Solid Tumors (RECIST) criteria. The primary endpoint was progression-free survival (PFS). The secondary end points included overall survival (OS), overall radiographic response rate (ORR), and safety. Twenty-nine patients were treated with pasireotide LAR (60 mg every 4 weeks) and 28 were evaluable for response. The median PFS was 11 months. The most favorable effect was observed in patients with low hepatic tumor burden, normal baseline chromogranin A, and high tumoral SSTR ${ }_{5}$ expression. Median OS has not been reached; the 30 -month OS rate was $70 \%$. The best radiographic response was partial response in one patient (4\%), stable disease in 17 patients $(60 \%)$, and progressive disease in ten patients $(36 \%)$.

Although grade 3/4 toxicities were rare, pasireotide LAR treatment was associated with a $79 \%$ rate of hyperglycemia including $14 \%$ grade 3 hyperglycemia. Although pasireotide appears to be an effective antiproliferative agent in the treatment of advanced NETs, the high incidence of hyperglycemia raises concerns regarding its suitability as a first-line systemic agent in unselected patients. SSTR ${ }_{5}$ expression is a potentially predictive biomarker for response.
\end{abstract}
Key Words
- pasireotide
- somatostatin analogs
- somatostatin receptors
- neuroendocrine tumors
- carcinoid

Endocrine-Related Cancer (2015) 22, 1-9

\section{Introduction}

Neuroendocrine tumors (NETs) are a heterogeneous group of neoplasms characterized by a relatively indolent rate of growth and a propensity to produce and secrete a variety of hormones that give rise to diverse clinical syndromes, including carcinoid syndrome. Based on their site of origin, NETs are often subclassified as pancreatic NETs (pNETs) and 
carcinoid tumors which are defined as NETs originating in the enterochromaffin cells of the airways and gastrointestinal tract (Kulke et al. 2012). In recent years, treatment options for metastatic NETs have expanded and targeted agents, including somatostatin analogs (SSAs), everolimus, and sunitinib, have entered into clinical use (Fazio et al. 2014).

Among the various emerging treatments, SSAs are associated with the most favorable side effect profile. Initially developed to palliate hormonal symptoms (Kvols et al. 1986), SSAs such as octreotide and lanreotide have also been shown to slow tumor progression in patients with advanced NETs (Faiss et al. 2003, Rinke et al. 2009). The randomized phase III PROMID trial evaluated octreotide long-acting repeatable (LAR) versus placebo in patients with metastatic midgut NETs and demonstrated a significant improvement in time-to-progression, thus defining a new paradigm in the treatment of small-intestinal NETs (Rinke et al. 2009). More recently, the CLARINET trial randomized patients with hormonally nonfunctioning gastroenteropancreatic NETs to receive depot-lanreotide versus placebo, also demonstrating a statistically significant improvement in median progression-free survival (PFS) from 18 months on placebo to unreached with lanreotide (Caplin et al. 2014). Tumor growth suppression is thought to be mediated through direct interaction between the SSA and its receptors on the tumor surface (direct effect) and also through inhibition of various circulating growth factors and cytokines such as insulin-like growth factor (indirect effect). It is still unclear which specific somatostatin receptor (SSTR) subtypes are primarily active in the antiproliferative effect of SSAs (Villaume et al. 2010, Sidéris et al. 2012).

Pasireotide is a novel multireceptor-targeted SSA with avid binding affinity to four of the five SSTR subtypes $\left(\mathrm{SSTR}_{1,2,3}\right.$ and $\mathrm{SSTR}_{5}$ ) (Schmid 2008). Compared with octreotide, pasireotide shows a 2.5 times lower binding affinity to $\mathrm{SSTR}_{2}$, but a 30-, five-, and 40-fold higher affinity for SSTR 1, SSTR $_{3}$, and SSTR 5 respectively (Schmid \& Schoeffter 2004). Results of preclinical studies have indicated that pasireotide reduces NET secretion and growth both in vitro and in vivo (van Hoek et al. 2009, Somnay et al. 2013), displaying an antiproliferative activity higher than octreotide, at least against the NCI-H727 bronchial carcinoid cell line (Ono et al. 2007). When tested in clinical trials, pasireotide has shown a favorable tolerability profile (Dietrich et al. 2012, Wolin et al. 2013b, Shenouda et al. 2014). Pasireotide has demonstrated superior efficacy over the current standard of care (octreotide LAR) in patients with acromegaly (Colao et al. 2014) and, based on the results of a phase III trial (Colao et al. 2012), has been approved in both Europe and the USA for the treatment of patients with Cushing's disease for whom surgery has failed or is not an option. Recently, in a phase II study enrolling patients with advanced NETs refractory or resistant to SSAs, s.c. pasireotide up to $1200 \mu \mathrm{g}$ twice daily was effective in controlling the symptoms of carcinoid syndrome in $27 \%$ of the cohort (Kvols et al. 2012).

Given the tolerable side effect profile of pasireotide LAR, its enhanced affinity for SSTRs, and its putative antiproliferative effects in patients with NETs, we conducted an open-label, single-arm, phase II study to investigate the antitumor activity of pasireotide LAR in treatment-naïve patients with metastatic pancreatic and extra-pancreatic NETs. The study also offered an opportunity to assess the correlation between SSTR staining pattern and clinical outcomes.

\section{Patients and methods}

\section{Patient selection}

This study was an open-label, single-arm, phase II prospective clinical trial. The protocol was approved by the Ethics Committee or the Institutional Review Board at each participating center. All patients provided written informed consent. The study was registered with clinicaltrials.gov (NCT01253161).

Eligible patients were required to be 18 years of age or older and have locally unresectable or metastatic grade 1 or 2 pancreatic or extra-pancreatic NETs. Mitotic count and ki-67 thresholds for the tumor grade index were based on the World Health Organization (WHO) 2010 classification (Rindi \& Arnold 2010). Patients with a tumoral ki-67 index of over $20 \%$ were excluded. Previous systemic antineoplastic treatment, including octreotide and lanreotide, was not permitted. Other key eligibility criteria were measurable disease by Response Evaluation Criteria in Solid Tumors (RECIST), minimum of 4 weeks since any major surgery, absolute neutrophil count of 1000 cells/ $\mu l$ or greater, platelets at a level of 75000 cells/ $\mu$ l or greater, hemoglobin over $8 \mathrm{~g} / \mathrm{dl}$, total bilirubin at or below 2 times the upper limit of normal (ULN), AST and ALT at or below 2 times ULN (less than 3 times ULN if liver metastases were present), creatinine at or below 2 times ULN, fasting serum cholesterol of $300 \mathrm{mg} / \mathrm{dl}$ or lower, and fasting serum triglycerides at or below 2.5 times ULN. Patients with uncontrolled diabetes mellitus or a fasting plasma glucose above 1.5 times ULN or HbA1c exceeding $8 \%$ were excluded, as were patients with symptomatic cholelithiasis. Other key exclusion criteria included a prolonged corrected QT interval (over $470 \mathrm{~ms}$ at screening) and a history of clinically significant cardiac arrhythmias.

Published by Bioscientifica Ltd. 


\section{Treatment and evaluation}

Pasireotide LAR (60 mg) was administered intramuscularly every 4 weeks. A dose reduction to $40 \mathrm{mg}$ every 4 weeks was allowed for patients unable to tolerate the full dose. All patients who developed hyperglycemia were required to monitor their fasting blood glucose levels using finger sticks twice daily and maintain a diary. Hyperglycemia was managed according to the investigators' judgment. Toxicities were graded according to the National Cancer Institute (NCI) Common Terminology Criteria for Adverse Events (CTCAE) version 4.0. Evaluation visits were scheduled every 4 weeks along with standard blood tests (complete blood count, comprehensive metabolic panel), while tumor markers such as chromogranin A (CgA) and other secretory hormones including 5-hydroxyindoleacetic acid (5-HIAA), gastrin, and glucagon were measured every 12 weeks, if elevated at baseline. Radiological assessment of tumor burden was performed by local radiologists who were blinded to patient clinical characteristics every 12 weeks using computed tomography (CT) or magnetic resonance imaging (MRI) scans and RECIST version 1.0 (Therasse et al. 2000) was used for evaluation of tumor response. Hepatic tumor load was quantified from CT/MRI scans by a semiquantitative three-dimensional approach (Rinke et al. 2009). Hepatic tumor burden was categorized as $0 \%$, between 0 and 10\% and more than 10\%.

\section{Immunohistochemical analysis of archival specimens}

Immunohistochemistry (IHC) was used to evaluate the expression of the SSTR subtypes SSTR ${ }_{1-5}$. Sections of 3-4 $\mu \mathrm{m}$ in thickness were cut from archival paraffin-embedded pathology specimens of accrued patients and subjected to an IHC staining protocol using the DakoCytomation Autostainer (DakoCytomation, Carpinteria, CA, USA). Microwave antigen retrieval with IHC Select EDTA buffer, pH 7.5 (Chemicon International, Temecula, CA, USA), was utilized. Endogenous peroxidase was blocked by hydrogen peroxide and blockage of avidin-binding protein was accomplished by using the Avidin-Biotin blocking kit (Vector Laboratories, Burlingame, CA, USA). The rabbit polyclonal primary antibodies against $\mathrm{SSTR}_{1-5}$ were provided by the University of Uppsala, Sweden. Dilutions and timing for the five SSTR antibodies were used as previously reported by Nasir et al. (2006). EnVision + HRP-labeled polymer antirabbit detection was used. Rabbit IgG was used for 60 min to replace the primary antibodies in the negative control sections. Pancreatic tissue was used as a positive control. The immunostained sections were examined under a light microscope by an experienced neuroendocrine pathologist (D Coppola) who was blinded to the patient clinical outcome. Immunoreactivity was scored into four categories, according to the intensity of the staining: $0,1+$, $2+$, and $3+$. The percentage of positive tumor cells was also scored semiquantitatively on a four-tiered scale: $0(0 \%)$, 1 (1-33\%), 2 (34-66\%), and 3 (67-100\%). The product of the intensity and the percentage scores determined the final score, which was classified as negative (score 0), weak (score 1-3), moderate (score 4-6), or strong (score 7-9). The correlation between SSTR staining patterns and clinical outcomes was analyzed in a descriptive fashion.

\section{Sample size calculation}

The primary efficacy endpoint was PFS. Secondary endpoints included overall survival (OS), overall radiographic response rates (ORR), and adverse events. The sample size calculation was based on the assumption that a true 1-year PFS rate of over $40 \%$ would be necessary to consider the investigational treatment sufficiently effective to pursue in further studies, whereas a true 1-year PFS of less than 19\% would not yield further interest in this agent. These PFS rates correspond to null and alternative hypothesis median PFS of 5 and 9 months respectively. The sample size was chosen based on a one-sided $\alpha$ level of $10 \%$ and a power of $90 \%$.

\section{Statistical analysis}

The Kaplan-Meier method was used to estimate all timeto-event functions. PFS was calculated from the date of first study treatment until the date of first detection of progressive disease or death due to any cause. OS was defined as the time from start of treatment until death as a result of any cause, with patients censored at the date of last follow-up if still alive. Exact 95\% CI were calculated for each proportion of interest. All tests were two sided and statistical significance was declared at $P<0.05$. Statistical analysis was performed using MedCalc statistical software 12.7 (MedCalc Software bvba, Ostend, Belgium). The assumption of exponential distribution was verified using the Hollander-Proschan test.

\section{Results}

\section{Patient population}

Demographic variables and clinical characteristics of 29 patients enrolled in the study are listed in Table 1. Median age of patients at study entry was 59 (31-75) years.

Published by Bioscientifica Ltd. 
Table 1 Patient demographics and clinical characteristics

\begin{tabular}{|c|c|c|}
\hline Characteristic & $\begin{array}{c}\text { No. of } \\
\text { patients }(=29)\end{array}$ & $\%$ \\
\hline \multicolumn{3}{|l|}{ Age (years) } \\
\hline Median & 59 & \\
\hline Range & $31-75$ & \\
\hline \multicolumn{3}{|l|}{ Gender } \\
\hline Male & 19 & 66 \\
\hline Female & 10 & 34 \\
\hline \multicolumn{3}{|l|}{ Race } \\
\hline White & 22 & 76 \\
\hline Black & 3 & 10 \\
\hline Hispanic & 3 & 10 \\
\hline Asian & 1 & 4 \\
\hline \multicolumn{3}{|c|}{ Performance status (ECOG) } \\
\hline 0 & 21 & 72 \\
\hline 1 & 8 & 28 \\
\hline \multicolumn{3}{|c|}{ Location of primary tumor } \\
\hline Small intestine & 14 & 49 \\
\hline Pancreas & 6 & 21 \\
\hline Rectum & 2 & 7 \\
\hline Appendix & 1 & 3 \\
\hline Lung & 1 & 3 \\
\hline Thymus & 1 & 3 \\
\hline Unknown & 4 & 14 \\
\hline \multicolumn{3}{|l|}{ Tumor grade } \\
\hline Low grade & 13 & 45 \\
\hline Intermediate grade & 16 & 55 \\
\hline \multicolumn{3}{|c|}{ Elevated baseline chromogranin A ( > ULN) } \\
\hline Yes & 18 & 62 \\
\hline No & 11 & 38 \\
\hline \multicolumn{3}{|c|}{ Baseline chromogranin $\mathrm{A}(\mathrm{nl}<15 \mathrm{ng} / \mathrm{ml})$} \\
\hline Median & 21 & \\
\hline Mean & 236 & \\
\hline Range & $1-2600$ & \\
\hline \multicolumn{3}{|c|}{ Elevated baseline 5 -HIAA } \\
\hline Yes & 9 & 31 \\
\hline No & 13 & 45 \\
\hline Unknown & 7 & 24 \\
\hline \multicolumn{3}{|l|}{ Liver involvement (\%) } \\
\hline 0 & 2 & 7 \\
\hline$<10$ & 12 & 41 \\
\hline$\geq 10$ & 15 & 52 \\
\hline \multicolumn{3}{|l|}{ Octreoscan positivity } \\
\hline No & 5 & 17 \\
\hline Yes & 21 & 73 \\
\hline Unspecified & 3 & 10 \\
\hline
\end{tabular}

Primary tumor sites included small intestine (14 patients), pancreas $(n=6)$, rectum $(n=2)$, unknown $(n=4)$, and other sites $(n=3)$. Thirteen patients had grade 1 tumors and 16 patients had grade 2 tumors. Twenty-seven patients had liver metastases, and hepatic tumor load was $\geq 10 \%$ in 15 patients $(52 \%)$. The two patients without liver involvement had lymph node metastases unresectable by surgery. Octreoscan was performed in 26 patients before study entry, and $73 \%$ were positive. Five patients had carcinoid syndrome. Printed in Great Britain

\section{Duration of therapy}

The intended total study duration was 24 months. Enrolled patients received a median of nine 28-day treatment cycles. Reasons for discontinuation included radiographic tumor progression (18), symptomatic decline (3), toxicity (2), and death on study (1). Five patients remained on the drug at the time of data analysis. One patient required a single-dose reduction, due to QTc prolongation. Hyperglycemia was the only toxicity leading to discontinuation of treatment (two patients).

\section{Progression-free and overall survival}

At the time of data cutoff, six patients had died and 23 patients were alive, with follow-up duration ranging from 5.8 to 34.6 months. As depicted in Fig. 1A, the overall median PFS was 11 months (95\% CI, 7.6-16 months). Stratified by tumor group (Fig. 1B), the median PFS of patients with midgut carcinoids and NETs of other primary sites was 14.8 months (95\% CI, 8.6-25.6 months) and 7.6 months (95\% CI, 4.5-12.7 months) respectively $(P=0.14)$. By grade (Fig. 1C), the median PFS of patients with grade 1 and 2 tumors was 13.4 months (95\% CI, 7.5-23.9 months) and 9.3 months respectively (95\% CI, 5.7-15.2 months; $P=0.43)$. Patients with high $(\geq 10 \%)$ hepatic tumor load had a significantly shorter PFS (7 months; 95\% CI, 4.4-11.3 months) compared with patients with low $(<10 \%)$ hepatic tumor burdens, who showed a median PFS of 17.8 months (95\% CI, 9.6-33.1 months; $P=0.05)$. Among patients with elevated baseline $\mathrm{CgA}$, those experiencing at least a 50\% reduction or normalization of the tumor marker within 3 months from the initiation of the treatment showed a median PFS of 11.8 months (95\% CI, 5.2-26.7 months), whereas among late and non-responders, the median PFS was 5.7 months (95\% CI, 3.3-9.9 months). Patients with normal baseline CgA exhibited a median PFS of 18.5 months (95\% CI, 9.4-36 months). There was no correlation between Octreoscan positivity and PFS $(P=0.55)$.

Median OS has not been reached. The 12-, 24-, and 30-month survival rates estimated by the Kaplan-Meier method were 89,70 , and $70 \%$ respectively (data not shown).

\section{Biochemical and radiological response}

Patients were followed for biochemical and radiological responses with CgA and 5-HIAA levels and imaging studies after every 12 weeks of treatment. Among 18 patients who

Published by Bioscientifica Ltd. 

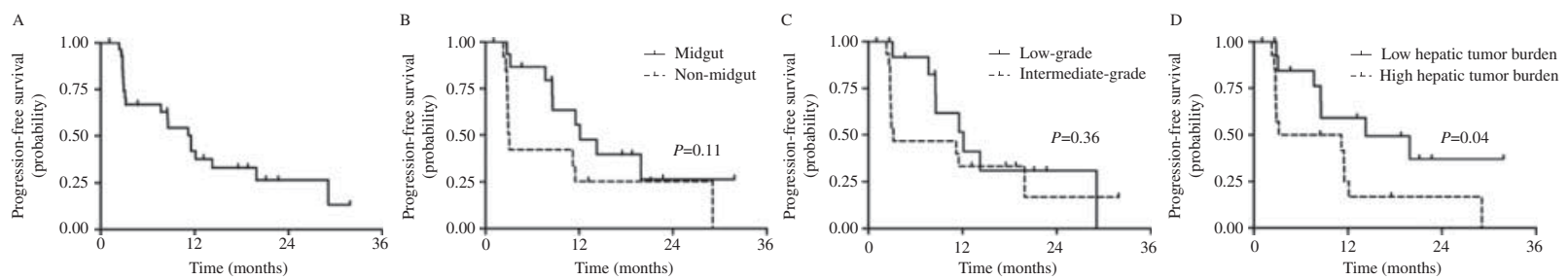

Figure 1

Kaplan-Meier estimate of progression-free survival (PFS). (A) For all 29 patients, 6 - and 12-month PFS rates were $66.9 \%( \pm 9)$ and $41.9 \%( \pm 9.9)$ respectively. (B) PFS by primary site, (C) PFS by tumor grade, and (D) PFS by hepatic tumor burden.

had baseline-elevated (above the ULN) serum CgA levels, eight patients (44\%) experienced major reductions (over $50 \%$ ) or normalization of the tumor marker. This biochemical response was always noted within 3 months of treatment initiation. Differences between the median baseline $\mathrm{CgA}$ concentration and its lowest values following initiation of treatment were statistically significant $(P=0.02)$. Best biochemical responses of CgA levels compared with baseline in patients with evaluable data are depicted in Fig. 2A. An insufficient number of patients had baseline elevations of 5-HIAA to allow reliable conclusions to be drawn regarding the 5-HIAA response.

Twenty-eight patients were assessable for radiological response. One patient withdrew from the study before radiographic evaluation due to toxicity (hyperglycemia). When best response to therapy was evaluated, $4 \%(1 / 28)$ of patients partially responded according to RECIST criteria, $60 \%(17 / 28)$ had stable disease, and 36\% (10/28) experienced progressive disease. The waterfall plot analysis showed some degree of tumor shrinkage in $46 \%$ $(13 / 28)$ of evaluable patients, while $54 \%(15 / 28)$ of the cohort appeared to experience continued tumor growth. Figure $2 \mathrm{~B}$ summarizes the maximum percentage changes from baseline in the sum of the longest diameters of target lesions. Of the five patients with carcinoid syndrome, four experienced an improvement of their symptoms.

\section{Safety profile}

All enrolled patients received treatment and were evaluated for toxicity. Pasireotide LAR was generally well tolerated with the exception of hyperglycemia. The side effects considered at least possibly related to the treatment are listed in Table 2. No drug-related grade 4 adverse events were observed on the trial, whereas grade 3 toxicities included cholecystitis (3.5\%) and hyperglycemia (14\%). Among the most common mild or moderate adverse events with a suspected study drug relationship were hyperglycemia $(65 \%)$, diarrhea (14\%), rash (7\%), fatigue (7\%), and myalgia (7\%). Hyperglycemia required intervention in 13 out of 22 patients and was managed with oral hypoglycemic agents or insulin in seven and six patients respectively. Despite discontinuation of pasireotide, three patients have remained on long-term insulin therapy. Only one of the 22 patients who experienced hyperglycemia during treatment with pasireotide had a
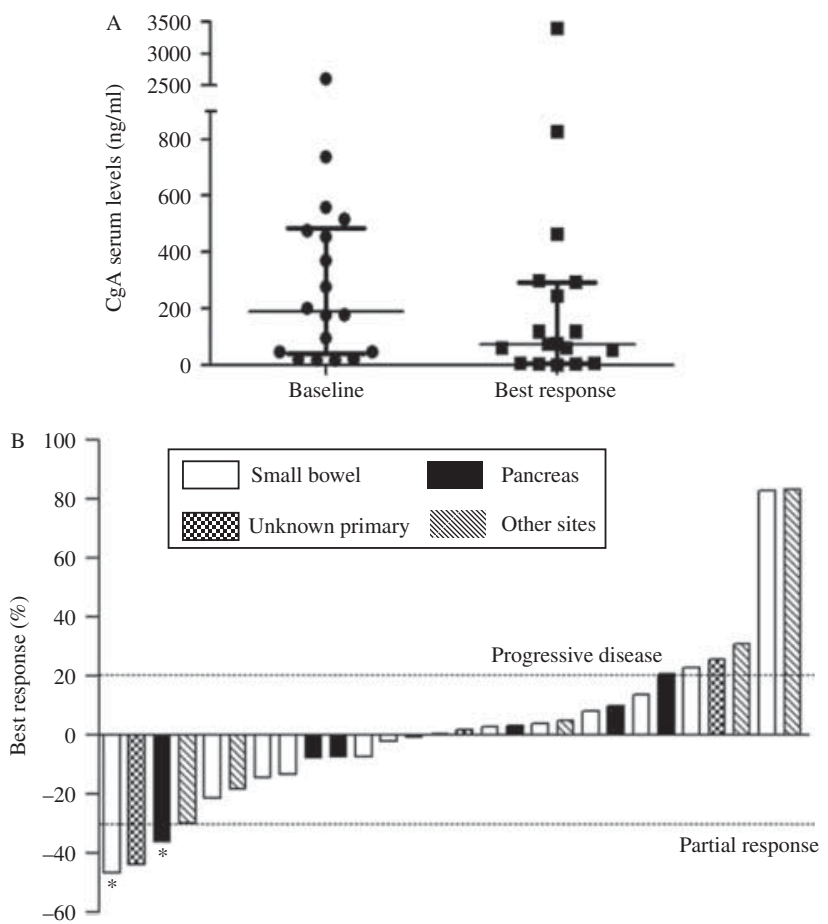

Evaluable patients $(n=28)$

Figure 2

Biochemical and radiological response following pasireotide treatment. (A) In patients with elevated baseline $\mathrm{CgA}$, median $\mathrm{CgA}$ concentrations decreased from 189 to $73.5 \mathrm{ng} / \mathrm{ml}$. The decrease was statistically significant $(P=0.02)$ according to the Wilcoxon-matched pairs signed rank test. Paired row values, median change, and interquartile range are represented. (B) Waterfall plot illustrating best radiographic response (percentage change) in each enrolled patient. *Decrease not confirmed at subsequent assessment, thus failing to match RECIST criteria for partial response.

Published by Bioscientifica Ltd. 
Table 2 Treatment-related toxicity

\begin{tabular}{|c|c|c|c|c|}
\hline & Grade 1 & Grade 2 & Grade 3 & Total \\
\hline \multicolumn{5}{|c|}{ Hematological toxicity, $n(\%)$} \\
\hline Anemia & $1(3)$ & $0(0)$ & $0(0)$ & $1(3)$ \\
\hline Thrombocytopenia & $1(3)$ & $0(0)$ & $0(0)$ & $1(3)$ \\
\hline \multicolumn{5}{|c|}{ Gastrointestinal toxicity (\%) } \\
\hline Diarrhea & $4(14)$ & $0(0)$ & $0(0)$ & $4(14)$ \\
\hline Nausea & $0(0)$ & $1(3)$ & $0(0)$ & $1(3)$ \\
\hline Vomiting & $0(0)$ & $1(3)$ & $0(0)$ & $1(3)$ \\
\hline Bloating & $1(3)$ & $0(0)$ & $0(0)$ & $1(3)$ \\
\hline Cholecystitis & $0(0)$ & $0(0)$ & $1(3)$ & $1(3)$ \\
\hline Mucositis & $1(3)$ & $0(0)$ & $0(0)$ & $0(0)$ \\
\hline Abdominal pain & & & & \\
\hline \multicolumn{5}{|l|}{ Cardiovascular toxicity (\%) } \\
\hline QT prolongation & $1(3)$ & $0(0)$ & $0(0)$ & $1(3)$ \\
\hline \multicolumn{5}{|l|}{ Skin toxicity (\%) } \\
\hline Rash & $2(7)$ & $0(0)$ & $0(0)$ & $2(7)$ \\
\hline \multicolumn{5}{|l|}{ Metabolism disorders (\%) } \\
\hline Hyperglycemia & $9(32)$ & $9(32)$ & $4(14)$ & $22(79)$ \\
\hline Hypoglycemia ${ }^{a}$ & $1(3)$ & $0(0)$ & $0(0)$ & $1(3)$ \\
\hline \multicolumn{5}{|l|}{ Miscellaneous } \\
\hline Fatigue & $2(7)$ & $0(0)$ & $0(0)$ & $2(7)$ \\
\hline Myalgia & $2(7)$ & $0(0)$ & $0(0)$ & $2(7)$ \\
\hline Hypertransaminasemia & $1(3)$ & $0(0)$ & $0(0)$ & $1(3)$ \\
\hline $\begin{array}{l}\text { Elevated alkaline } \\
\text { phosphatase }\end{array}$ & $1(3)$ & $0(0)$ & $0(0)$ & $1(3)$ \\
\hline
\end{tabular}

${ }^{\text {a }}$ Hypoglycemia that occurred during an episode of bowel obstruction in an insulin-treated patient.

medical history of diabetes at baseline. Mean fasting glucose levels increased from $106 \mathrm{mg} / \mathrm{dl}$ at baseline to $150 \mathrm{mg} / \mathrm{dl}$ at the end of the study.

\section{Tissue analysis}

Archival pathology specimens were available for 23 patients. Eight specimens were obtained from the primary tumor location and 15 were from liver metastases. As summarized in Table 3, the median primary tumor size was $2 \mathrm{~cm}(0.4-$ $3.5)$. Necrosis was present in $4 \%(1 / 23)$ of specimens and vascular invasion in $30 \%(7 / 23)$. When specimens were immunostained using antibodies to $\mathrm{SSTR}_{1-5}$, the median IHC intensity score was four for $\mathrm{SSTR}_{2}$ (range $0-9$ ) and $\mathrm{SSTR}_{3}$ (range 2-9), three for $\mathrm{SSTR}_{4}$ (range 1-9), five for $\mathrm{SSTR}_{1}$ (range

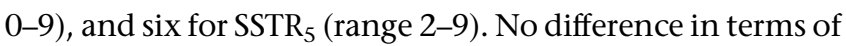
median intensity of immunostaining was observed after stratification for tumor site (primary/hepatic metastasis; $P=0.68$ ), primary tumor origin (midgut/non-midgut; $P=0.73)$, and grade $(P=0.7)$. The pattern of immunostaining was predominantly diffuse (cytoplasmic and nuclear) for $\mathrm{SSTR}_{1}$, whereas the expression of the other SSTR subtypes was mainly cytoplasmic (Fig. 3).

Univariate analyses revealed that only high $\mathrm{SSTR}_{5}$ expression was associated with prolonged PFS $(P=0.0068)$.
The median PFS for patients with tumors expressing low levels of $\mathrm{SSTR}_{5}$ was 2.8 months (95\% CI, 2.3-12.1 months) compared with 19.9 months (95\% CI, 7.7-29.1 months) among the patients expressing high levels of SSTR . There $^{2}$ was no significant correlation between expression of $\mathrm{SSTR}_{5}$ and the proliferative activity by ki-67 $(P=0.52)$. After stratification for median expression of $\mathrm{SSTR}_{1-5}$, the median PFS was 14.3 months (95\% CI, 3.2-29.1 months) and 5.8 months (95\% CI, 2.8-12.1 months) in the population showing median high or low intensity of SSTR expression respectively $(P=0.13)$.

\section{Discussion}

SSAs have an established place in the medical treatment of patients with NETs, being the standard of care for symptom control in patients with functional, hormonesecreting neoplasms. Moreover, both the PROMID (Rinke et al. 2009) and CLARINET (Caplin et al. 2014)

Table 3 Pathological characteristics of the patient population

\begin{tabular}{|c|c|c|}
\hline Characteristic & No. of patients $(=23)$ & $\%$ \\
\hline \multicolumn{3}{|l|}{ Primary tumor size $(\mathrm{cm})$} \\
\hline Median & 2 & \\
\hline Range & $0.4-3.5$ & \\
\hline \multicolumn{3}{|l|}{ Tumor necrosis } \\
\hline Present & 1 & 4 \\
\hline Absent & 12 & 52 \\
\hline Unknown & 10 & 44 \\
\hline \multicolumn{3}{|l|}{ Angioinvasion } \\
\hline Present & 7 & 30 \\
\hline Absent & 16 & 70 \\
\hline \multicolumn{3}{|l|}{ Ki-67 (\%) } \\
\hline Median & 5 & \\
\hline Range & $1-20$ & \\
\hline \multicolumn{3}{|l|}{ SSTR expression } \\
\hline \multicolumn{3}{|l|}{ SSTR $_{1}$} \\
\hline Low (negative/weak) & $2 / 5$ & $8 / 22$ \\
\hline High (moderate/strong) & $9 / 6$ & $39 / 26$ \\
\hline Unknown & 1 & 4 \\
\hline \multicolumn{3}{|l|}{$\mathrm{SSTR}_{2}$} \\
\hline Low (negative/weak) & $1 / 8$ & $4 / 35$ \\
\hline High (moderate/strong) & $12 / 1$ & $52 / 4$ \\
\hline Unknown & 1 & 4 \\
\hline \multicolumn{3}{|l|}{$\mathrm{SSTR}_{3}$} \\
\hline Low (negative/weak) & $0 / 7$ & $0 / 30$ \\
\hline High (moderate/strong) & $13 / 2$ & $57 / 8$ \\
\hline Unknown & 1 & 4 \\
\hline \multicolumn{3}{|l|}{$\mathrm{SSTR}_{4}$} \\
\hline Low (negative/weak) & $0 / 16$ & $0 / 69$ \\
\hline High (moderate/strong) & $5 / 1$ & $22 / 4$ \\
\hline Unknown & 1 & 4.5 \\
\hline \multicolumn{3}{|l|}{$\mathrm{SSTR}_{5}$} \\
\hline Low (negative/weak) & $0 / 5$ & $0 / 22$ \\
\hline High (moderate/strong) & $14 / 3$ & $61 / 13$ \\
\hline Unknown & 1 & 4 \\
\hline
\end{tabular}

Published by Bioscientifica Ltd. 

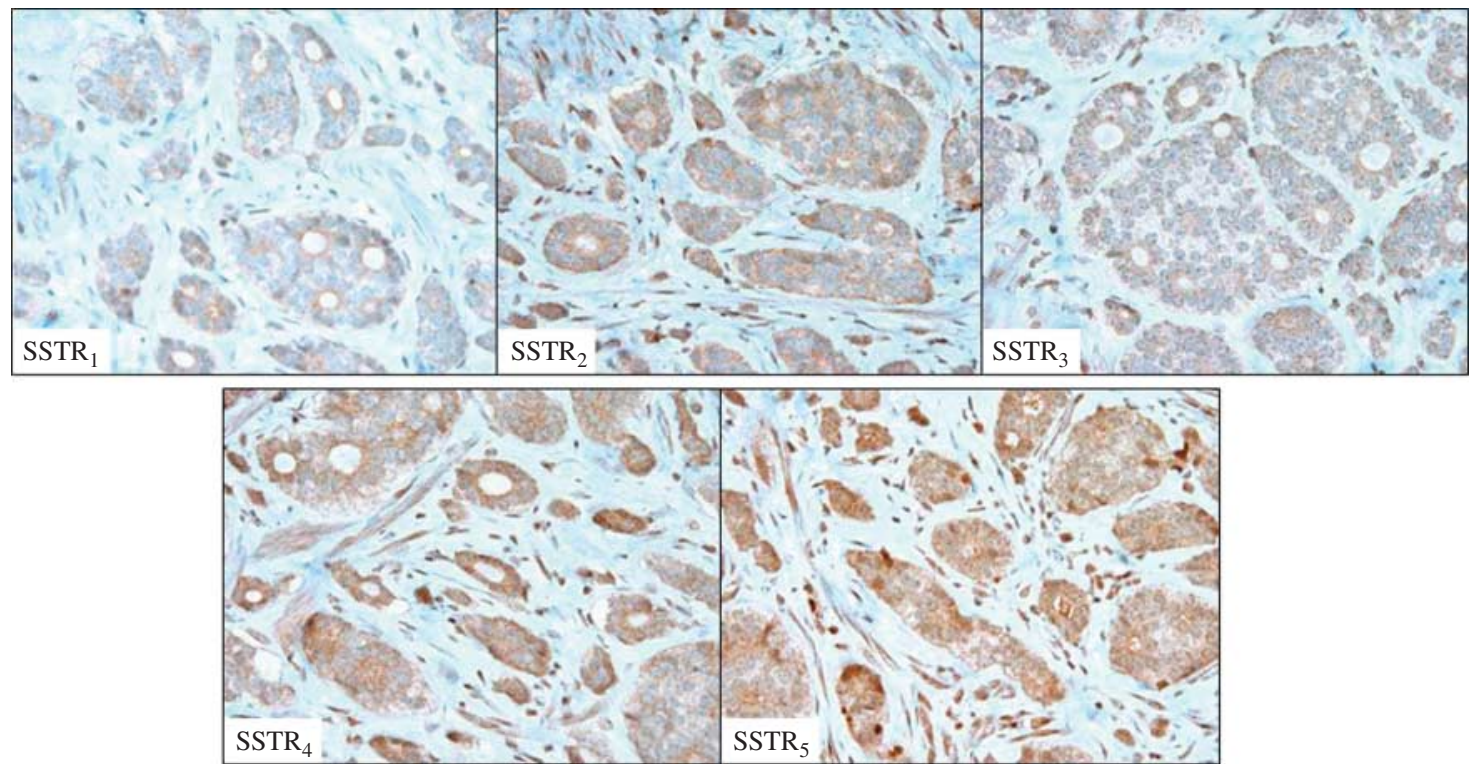

Figure 3

Examples of positive immunohistochemical staining for $\mathrm{SSTR}_{1-5}$ subtypes (magnification: $\times 40$ ). A full colour version of this figure is available at http://dx.doi. org/10.1530/ERC-14-0360.

studies have recently provided compelling evidence that SSAs can inhibit tumor growth in patients with metastatic well-differentiated NETs of the gastroenteropancreatic tract. Whereas both octreotide and lanreotide primarily target SSTR $_{2}$, pasireotide targets four of the five SSTRs and theoretically represents a more potent antiproliferative agent (Schmid \& Schoeffter 2004).

As a practical matter, assessing the efficacy of an antiproliferative agent in a single-arm phase II study is quite difficult, particularly in a very heterogeneous cohort of patients. Our assumption was that median PFS in the absence of treatment would be approximately 5 months (null hypothesis) whereas a median study PFS of longer than 9 months would indicate that pasireotide had significant activity. These assumptions were based on data sets from several clinical trials, including the placebo arms of the PROMID study (median PFS 6 months) (Rinke et al. 2009), and the RADIANT 3 trial in pNETs (median PFS 4.6 months on local review) (Yao et al. 2011). Also, we did not expect to achieve as long a median PFS as was seen in the treatment arm of the PROMID study (14.3 months) given the more aggressive tumor types eligible for our trial. With these previous study results in mind, the median PFS of 11 months observed in our study is encouraging and indicates that pasireotide LAR is an active antiproliferative agent capable of inhibiting tumor growth in a heterogeneous population of NETs.
Our trial patients were monitored carefully for emergence of hyperglycemia, and a high rate of elevated fasting blood glucose was observed. Although the majority of cases were mild, $45 \%$ of trial patients began medical treatment for hyperglycemia, nearly half of whom required insulin. Three patients remained on insulin for over a year after discontinuation of pasireotide. In contrast with results reported previously (Kvols et al. 2012), no correlation between a medical history of diabetes or impaired glucose tolerance at study entry and pasireotide-induced hyperglycemia was seen. The high rate of hyperglycemia observed in this trial raises questions regarding the suitability of pasireotide as a first-line treatment agent in NETs, particularly compared with octreotide or lanreotide, which are lacking in significant toxicities.

A potential role for pasireotide in NETs may exist in the salvage setting where the risk:benefit ratio may be more favorable. Indeed, results of a phase III study of pasireotide versus high-dose octreotide among patients with refractory carcinoid syndrome were reported recently (Wolin et al. 2013a). Although, in this study, pasireotide treatment did not achieve the primary endpoint of improvement in carcinoid syndrome, it was associated with a statistically significant prolongation in PFS (exploratory endpoint) compared with octreotide. Future studies that preselect tumors based on SSTR profile may identify patients more likely to respond to pasireotide than to the currently

Published by Bioscientifica Ltd. 
available SSAs. Our results indicate that $\mathrm{SSTR}_{5}$-expressing tumors may be particularly sensitive to pasireotide therapy.

\section{Conclusion}

Pasireotide LAR appears to significantly inhibit growth of NETs; however, it remains unclear whether its antiproliferative effect is superior to those of the currently available SSAs octreotide and lanreotide, and whether targeting multiple SSTRs results in improved tumor control in unselected patients. The high rate of hyperglycemia requiring intervention represents a potential impediment to the use of pasireotide as a first-line agent. Future studies should focus on the evaluation of pasireotide in patients progressing on octreotide or lanreotide. Expression of $\mathrm{SSTR}_{5}$ is a potential predictive biomarker for response.

\section{Declaration of interest}

Dr J R Strosberg has performed consultation for Novartis within institutional conflict of interest payment guidelines. All remaining authors have declared no conflicts of interest.

\section{Funding}

This work was supported by Novartis, Inc.

\section{References}

Caplin ME, Pavel M, Ćwikła JB, Phan AT, Raderer M, Sedláčková E, Cadiot G, Wolin EM, Capdevila J, Wall L et al. 2014 Lanreotide in metastatic enteropancreatic neuroendocrine tumors. New England Journal of Medicine 371 224-233. (doi:10.1056/NEJMoa1316158)

Colao A, Petersenn S, Newell-Price J, Findling JW, Gu F, Maldonado M, Schoenherr U, Mills D, Salgado LR, Biller BM et al. 2012 A 12-month phase 3 study of pasireotide in Cushing's disease. New England Journal of Medicine 366 914-924. (doi:10.1056/NEJMoa1105743)

Colao A, Bronstein MD, Freda P, Gu F, Shen CC, Gadelha M, Fleseriu M, van der Lely AJ, Farrall AJ, Hermosillo Reséndiz K et al. 2014 Pasireotide versus octreotide in acromegaly: a head-to-head superiority study. Journal of Clinical Endocrinology and Metabolism 99 791-799.

Dietrich H, Hu K, Ruffin M, Song D, Bouillaud E, Wang Y \& Hasskarl J 2012 Safety, tolerability, and pharmacokinetics of a single dose of pasireotide long-acting release in healthy volunteers: a single-center Phase I study. European Journal of Endocrinology 166 821-828. (doi:10.1530/EJE-11-0773)

Faiss S, Pape UF, Böhmig M, Dörffel Y, Mansmann U, Golder W, Riecken EO, Wiedenmann B \& International Lanreotide and Interferon Alfa Study Group 2003 Prospective, randomized, multicenter trial on the antiproliferative effect of lanreotide, interferon alfa, and their combination for therapy of metastatic neuroendocrine gastroenteropancreatic tumors - the International Lanreotide and Interferon Alfa Study Group. Journal of Clinical Oncology 21 2689-2696. (doi:10.1200/JCO. 2003.12.142)

Fazio N, Scarpa A \& Falconi M 2014 Molecular targeted therapy in enteropancreatic neuroendocrine tumors: from biology to clinical practice. Current Medicinal Chemistry 21 1017-1025. (doi:10.2174/ 09298673113209990237) van Hoek M, Hofland LJ, de Rijke YB, van Nederveen FH, de Krijger RR, van Koetseveld PM, Lamberts SW, van der Lely AJ, de Herder WW \& Feelders RA 2009 Effects of somatostatin analogs on a growth hormonereleasing hormone secreting bronchial carcinoid, in vivo and in vitro studies. Journal of Clinical Endocrinology and Metabolism 94 428-433. (doi:10.1210/jc.2008-1712)

Kulke MH, Benson AB III, Bergsland E, Berlin JD, Blaszkowsky LS, Choti MA, Clark OH, Doherty GM, Eason J, Emerson L et al. 2012 Neuroendocrine tumors. Journal of the National Comprehensive Cancer Network 10 724-764.

Kvols LK, Moertel CG, O'Connell MJ, Schutt AJ, Rubin J \& Hahn RG 1986 Treatment of the malignant carcinoid syndrome. Evaluation of a long-acting somatostatin analogue. New England Journal of Medicine 315 663-666. (doi:10.1056/NEJM198609113151102)

Kvols LK, Oberg KE, O’Dorisio TM, Mohideen P, de Herder WW, Arnold R, Hu K, Zhang Y, Hughes G, Anthony L et al. 2012 Pasireotide (SOM230) shows efficacy and tolerability in the treatment of patients with advanced neuroendocrine tumors refractory or resistant to octreotide LAR: results from a phase II study. Endocrine-Related Cancer 19 657-666. (doi:10.1530/ERC-11-0367)

Nasir A, Stridsberg M, Strosberg J, Su PH, Livingston S, Malik HA, Kelley ST, Centeno BA, Coppola D, Malafa ME et al. 2006 Somatostatin receptor profiling in hepatic metastases from small intestinal and pancreatic neuroendocrine neoplasms: immunohistochemical approach with potential clinical utility. Cancer Control 13 52-60.

Ono K, Suzuki T, Miki Y, Taniyama Y, Nakamura Y, Noda Y, Watanabe M \& Sasano H 2007 Somatostatin receptor subtypes in human nonfunctioning neuroendocrine tumors and effects of somatostatin analogue SOM230 on cell proliferation in cell line NCI-H727. Anticancer Research 27 2231-2239.

Rindi G \& Arnold R 2010 Nomenclature and classification of neuroendocrine neoplasms of the digestive system. In World Health Organization Classification of Tumours of the Digestive System, pp 13-14. Eds F Bosman, F Carneiro, R Hruban \& N Theise. Lyon, France: IARC Press.

Rinke A, Müller HH, Schade-Brittinger C, Klose KJ, Barth P, Wied M, Mayer C, Aminossadati B, Pape UF, Bläker M et al. 2009 Placebo-controlled, double-blind, prospective, randomized study on the effect of octreotide LAR in the control of tumor growth in patients with metastatic neuroendocrine midgut tumors: a report from the PROMID Study Group. Journal of Clinical Oncology 27 4656-4663. (doi:10.1200/JCO. 2009.22.8510)

Schmid HA 2008 Pasireotide (SOM230): development, mechanism of action and potential applications. Molecular and Cellular Endocrinology 286 69-74. (doi:10.1016/j.mce.2007.09.006)

Schmid HA \& Schoeffter P 2004 Functional activity of the multiligand analog SOM230 at human recombinant somatostatin receptor subtypes supports its usefulness in neuroendocrine tumors. Neuroendocrinology 80 (suppl 1) S47-S50. (doi:10.1159/000080741)

Shenouda M, Maldonado M, Wang Y, Bouillaud E, Hudson M, Nesheiwat D \& Hu K 2014 An open-label dose-escalation study of once-daily and twice-daily pasireotide in healthy volunteers: safety, tolerability, and effects on glucose, insulin, and glucagon levels. American Journal of Therapeutics 21 164-173. (doi:10.1097/MJT.0b013e31824c3eb4)

Sidéris L, Dubé P \& Rinke A 2012 Antitumor effects of somatostatin analogs in neuroendocrine tumors. Oncologist 17 747-755. (doi:10.1634/ theoncologist.2011-0458)

Somnay Y, Chen H \& Kunnimalaiyaan M 2013 Synergistic effect of pasireotide and teriflunomide in carcinoids in vitro. Neuroendocrinology 97 183-192. (doi:10.1159/000341810)

Therasse P, Arbuck SG, Eisenhauer EA, Wanders J, Kaplan RS, Rubinstein L, VerweijJ, Van Glabbeke M, van Oosterom AT, Christian MC et al. $2000 \mathrm{New}$ guidelines to evaluate the response to treatment in solid tumors. European Organization for Research and Treatment of Cancer, National Cancer Institute of the United States, National Cancer Institute of Canada. Journal of the National Cancer Institute 92 205-216. (doi:10.1093/jnci/92.3.205)

Villaume K, Blanc M, Gouysse G, Walter T, Couderc C, Nejjari M, Vercherat C, Cordier-Bussat M, Roche C \& Scoazec JY 2010 
VEGF secretion by neuroendocrine tumor cells is inhibited by octreotide and by inhibitors of the PI3K/AKT/mTOR pathway. Neuroendocrinology 91 268-278. (doi:10.1159/000289569)

Wolin EM, Jarzab B, Eriksson B, Walter T, Toumpanakis C, Morse M, Tomassetti P, Weber M, Fogelman DR, Ramage J et al. 2013a A multicenter, randomized, blinded, phase III study of pasireotide LAR versus octreotide LAR in patients with metastatic neuroendocrine tumors (NET) with disease-related symptoms inadequately controlled by somatostatin analogs. Journal of Clinical Oncology 31 (suppl; abstr 4031) 15s.
Wolin EM, Hu K, Hughes G, Bouillaud E, Giannone V \& Resendiz KH $2013 b$ Safety, tolerability, pharmacokinetics, and pharmacodynamics of a longacting release (LAR) formulation of pasireotide (SOM230) in patients with gastroenteropancreatic neuroendocrine tumors: results from a randomized, multicenter, open-label, phase I study. Cancer Chemotherapy and Pharmacology 72 387-395. (doi:10.1007/s00280-013-2202-1)

Yao JC, Shah MH, Ito T, Bohas CL, Wolin EM, Van Cutsem E, Hobday TJ, Okusaka T, Capdevila J, de Vries EG et al. 2011 Everolimus for advanced pancreatic neuroendocrine tumors. New England Journal of Medicine 364 514-523. (doi:10.1056/NEJMoa1009290)

Received in final form 23 September 2014

Accepted 6 November 2014

Made available online as an Accepted Preprint

6 November 2014
Published by Bioscientifica Ltd. 